\title{
Implications for space-based UHECR observation from UV background light measurements by JEM-EUSO pathfinders
}

\author{
K. Shinozaki, M. Bertaina \\ Department of Physics, University of Torino, Via P. Giuria, 1, I-10125 Turin, Italy \\ INFN-Torino, Via P. Giuria, 1, I-10125 Turin, Italy \\ E-mail: kenjikry@riken.jp \\ P. Bobík*, Š. Mackovjak, M. Putiš \\ Department of Space Physics, Institute of Experimental Physics, Slovak Academy of Science, \\ Watsonova 47, SK-040 01 Košce, Slovakia
}

for the JEM-EUSO Collaboration

EUSO (Extreme Universe Space Observatory) is a next-generation space-based UHECR observation concept to detect extensive air shower events by the fluorescence technique. The determination of the exposure for UHECR observations is essential to reach the scientific objectives. It involves trigger efficiency, observational duty cycle, impacts of clouds etc. UV light from the terrestrial and extraterrestrial sources is a major background for UHECR observations. Such background includes man-made light, airglow emission starlight, moonlight, zodiacal light etc. In the EUSO pathfinder missions, we carried out the measurements of the UV light by operating a prototype $1 \mathrm{~m}$ square refractive Fresnel telescope with a photo-detector module on balloons and on the ground. So far, flights of EUSO-Balloon and EUSO-SPB (Supper Pressure Balloon) achieved 1- and 12-night flights in Canada and south Pacific, respectively. Using different optics, two satellite-borne pathfinders, TUS (Tracking Ultraviolet Set-up) has been operational and Mini-EUSO are currently scheduled. On the ground EUSO-TA has been operational at the Telescope Array experiment site. All the pathfinders are designed to measure UV light to provide a larger amount of data in various conditions. In the present contribution, the basic concept to establish a scheme to determine the exposure for space-based UHECRs is discussed. A part of results from EUSO-Balloon about the background light measurements relevant to the exposure determination are presented. We also introduce an outlook for further studies by EUSO-SPB, TUS and Mini-EUSO.

35th International Cosmic Ray Conference - ICRC2017

10-20 July, 2017

Bexco, Busan, Korea

* Speaker. 


\section{Introduction}

The origin and production mechanisms of ultra-high energy cosmic rays (UHECRs) with energies, $E_{0} \gtrsim 4 \times 10^{19} \mathrm{eV}$, are unsolved problems in contemporary astroparticle physics. UHECRs have been observed through indirect measurements of air showers by detecting coincident secondary particles or UV fluorescence light induced by those particle cascade. Observations have been carried out using particle detectors, commonly called surface detectors (SDs) and/or UV-sensitive telescopes, i.e. fluorescence detectors (FDs). They are operated to detect signals from air showers above the background: against secondary particles from lower energy cosmic rays for SDs and against night sky background light for FDs.

Today, using both SDs and FDs on the ground, air shower experiments with observation area $S_{\text {obs }}$ beyond a few thousand square kilometers, such as the Pierre Auger Observatory [1], the Telescope Array [2] and its extension, TA $\times 4$ [3] are being operated or planned. For scientific interpretations of the observed data, the fluxes of UHECRs are most relevant to determine two major outputs from the UHECR experiments, namely the energy spectrum and arrival direction distribution on the Celestial Sphere. The former is the flux as a function of energy. The latter represents the distribution of the flux, usually above a given energy cut, on the celestial coordinates.

So far, however, the extremely low flux, $\sim 1$ UHECR or fewer in $1 \mathrm{~km}^{2}$ per century has prevented further advancements for understanding their nature, and in particular on for the highest energy cosmic rays above $10^{20} \mathrm{eV}$. To overcome such a difficulty, a drastic enhancement of the exposure for the UHECR observations is essential and the observation from a space-based platform is a promising option.

The JEM-EUSO (Extreme Universe Space Observatory on-board the Japanese Experiment Module) collaboration has been promoting the realization of a wide field-of-view (FOV) fluorescence telescope operating from Space [4]. Looking to the nadir from a few-hundred-kilometer above sea level, such a telescope covers a few orders of magnitude lager $S_{\text {obs }}$ area than ground-based air shower arrays. Despite of several conceptual advantages, the fluorescence technique has never been realized in Space. To demonstrate the key technologies developed for JEM-EUSO, we planed a few pathfinder missions on balloon, satellite and on the ground and a few have been carried out.

Depending on the studied designs of the missions [5, 6], EUSO-type instruments employ a few-meter-diameter optics, either refractive or reflective, and focal surface detectors consisting of multi-anode photomultiplier tubes (MAPMTs). The photo-detector module (PDM) consisting of 36 MAMPTs are designed to perform single photon counting over 2304 pixels in total with an integration time of $\sim 2.5 \mu \mathrm{s}$ [7]. This duration was chosen to fit a shortest traverse time of light in the atmosphere that crosses the angular size seen by a pixel in the original JEM-EUSO design as a result of optimization of the air shower observation from the Space.

For both ground-based and space-based FDs, air showers are detected against the background light. This light seen on the Earth side, instead of the night sky background, affects the trigger efficiency of the data acquisition in the orbit and the data analysis on the ground. In the present contribution, we focus on the first point, in particular about the effect to the exposure of UHECR observations. The basic concept of the exposure determination for space-based UHECR observations is summarized together with a part of EUSO-Balloon measurements. 


\section{EUSO pathfinders}

So far, two balloon-borne pathfinder missions, EUSO-Balloon and EUSO-SPB, have been completed. Now other two, EUSO-TA and TUS, are operational. Mini-EUSO is scheduled to be operated. Each pathfinder measures the UV background light from different viewpoint as summarized below.

TUS (Tracking Ultaviolet Set-up) $[8,9,10]$ is a pathfinder mission. Launched on April 28, 2016, it has been operated on the Lomonosov satellite in the orbit at $\sim 500 \mathrm{~km}$ altitude. Using a 2 $\mathrm{m}^{2}$ reflective Fresnel optics with 256 photomultiplier tubes, covering a $9^{\circ} \mathrm{FOV}$. With an inclination of $\sim 97^{\circ}$ it has potential to measure the background light above most part of the Earth.

Mini-EUSO [11] is a $25 \mathrm{~cm}$ scale telescope with a refractive Fresnel optics mounted on the UVtransparent window of the Russian module Zvezda in the International Space Station at $\sim 400 \mathrm{~km}$ altitude. According to the orbit inclination, Mini-EUSO has the potential to map within the latitudes of $\pm 51.6^{\circ}$. A prototype PDM will cover a $\sim 45^{\circ}$ square FOV along with a delegated photo-detector providing for stand-alone UV light measurements.

EUSO-Balloon was carried out its stratospheric flight in August 2014 from Timmins, Canada. EUSO-Balloon reached a float altitude of $\sim 38 \mathrm{~km}$ [12]. Using a Fresnel refractive optics consisting of two $1 \mathrm{~m}$ square lenses with a PDM, we performed UV background light measurements. By analyzing a $\sim 2.5$ hour data, we obtained UV image of night-time earth in Timmins and neighboring areas [13, 14]. In the present and other contributions [15], we present a part of results.

EUSO-SPB [16] on the NASA's Super Pressure Balloon (SPB) [17] was launched from Wanaka, New Zeeland on April 24, 2017 UTC. The instrument was similar to that of EUSO-Balloon but with the upgraded subsystems such as the autonomous first level trigger algorithm as developed for JEM-EUSO [18]. Due to the gas leakage of the balloon envelope, the operation of EUSO-SPB was terminated on May 7 UTC. Preliminary results are presented in these proceedings $[16,19]$. Depending on night, the altitude of operations varied in $\sim 16-30 \mathrm{~km}$. The data analysis of EUSOSPB, including of the UV background light, is in progress. Thanks to the opportunity of a long duration flight and the trigger system, EUSO-SPB had a potential to detect air showers in a few times of $10^{18} \mathrm{eV}$ regime from above the ground by fluorescence technique [20].

A ground-based pathfinder experiment 'EUSO-TA' [21, 22] is also in operational at the site of the TA experiment in collaboration with the TA group. It measures the night sky background, including direct airglow emission. So far, at least four air shower events have been detected with confidence in coincidence with external trigger provided by TA-FDs [23].

\section{Exposure of UEHCR observations}

For UHECR observations, the differential flux $J\left(E_{0}\right)$ is given as follows:

$$
N_{\text {eve }}\left(E_{0}, E_{0}+\Delta E_{0}\right)=A\left(E_{0}\right) \cdot J\left(E_{0}\right)
$$

where $N_{\text {eve }}\left(E_{0}, E_{0}+\Delta E_{0}\right)$ is the number of observed UHECR events with energies between $E_{0}$ and $E_{0}+\Delta E_{0}$ and $A\left(E_{0}\right)$ is the exposure of the experiment to UHECRs at the energy $E_{0}$. The exposure is obtained by the integral of the instantaneous aperture, $\dot{A}$, over the observation time as follows:

$$
A\left(E_{0}\right) \equiv \int \dot{A}\left(E_{0}, t\right) d t
$$


Within the uncertainty and error in energy estimation, $N_{\text {eve }}$ is given by the experiment, while the exposure needs to be separately estimated. For the air shower array, $S_{\text {obs }}$ is well confined by the boundary SDs of the array and thus nearly constant $\dot{A}$ is expected in the energy of interest. For the FDs, the instantaneous aperture depends on energy in both ground- and space-based observations.

In the latter case, the variation of UV background light would be an important factor since the background conditions largely vary along the orbit. For EUSO-type observatories, the thresholds applied to the trigger algorithms are dynamically set by the average or maximum count rate by background within the operational unit, eg. PDM or the elementary cells, independent units of four MAPMTs in the PDM [19, 24, 25]. It is needed to comply the limited telemetry available for the mission, whilst efficiently discriminating the real real from triggers due to background fluctuations.

Under the moonless and clear atmosphere, considered to be the lowest background light conditions, a reference count rate $n_{0}$ can be defined as the average count rate. The corresponding reference function of the instantaneous aperture $\dot{A}_{0}$ for UHECR observations is obtained by simulating a large number of air showers and the instrument response. The instantaneous aperture $\dot{A}$ empirically scales to variable $n$ as follows $[24,26]$ :

$$
\dot{A}\left(E_{0} ; n\right)=\dot{A}_{0}\left(\sqrt{\frac{n_{0}}{n}} \cdot E_{0}\right)
$$

in units of $\mathrm{km}^{2} \mathrm{sr}$, here neglecting the effects of clouds and the local light component. This equation represents the energy scale for the trigger efficiency following to the signal-to-noise ratio as the trigger algorithms have been designed to keep a constant background trigger rate [18].

$n$ is variable over time. By integrating Equation (3.3) over the mission lifetime $T_{0}$, the exposure $A$ is given as a function of energy as follows:

$$
\begin{aligned}
A\left(E_{0}\right) & \equiv \int_{0}^{T_{0}} \dot{A}\left(E_{0}, n(t)\right) d t \\
& =\int_{0}^{n} \lim \left[\dot{A}_{0}\left(\sqrt{\frac{n_{0}}{n}} \cdot E_{0}\right) \cdot\left(\frac{\Delta t}{\Delta n}\right)\right] d n .
\end{aligned}
$$

in units of $\mathrm{km}^{2}$ sr yr. $\dot{A}=0$ for the time intervals when no UHECR observation, eg. during the day, is undertaken, including the case of $n>n_{\text {lim }}$ such as intense back-ground moonlight.

For UHECRs with $E_{0} \gtrsim 10^{20} \mathrm{eV}$, JEM-EUSO has a nearly constant geometrical aperture [24, 26]. Taking into account the effects of the clouds and the local light, the overall exposure at the highest energies can be expressed as:

$$
A(\infty) \approx \dot{A}_{0}(\infty) \cdot \kappa_{\mathrm{C}} \cdot \eta \cdot\left(1-f_{\mathrm{loc}}\right) \cdot T_{0},
$$

where $\kappa_{C}$ is the cloud efficiency, $\eta$ is the observational duty cycle, defined as a ratio of the observation time $T_{\mathrm{obs}}$ to the whole mission lifetime $T_{0}$ and $f_{\text {loc }}$ is the fraction of the area with intense light. $\kappa_{\mathrm{C}}$ describes the ratio of the aperture taking into account the presence and distribution of cloud types to the one for the clear atmosphere conditions [24, 26, 27].

\section{UV light measurements by EUSO pathfinders}

As represented in Equation (3.3), the background plays a role to determine the aperture in lower energy range. The background level $n(t)$ as a function of time will be a key parameter 
to determine the overall exposure that allows the calculation of Equation (3.5). At the highest energies, the background level is a limiting factor of $T_{\mathrm{obs}}$.

In previous articles $[24,26]$, we evaluated the expected exposure to UHECR observations by the JEM-EUSO mission. As the moonlight is the largest source of the $n$ variation, we evaluated $\eta$ taken into account phase of the Moon and back-scattered moonlight intensity in the orbit. The fraction, $f_{\text {loc }}$, of the area with intense light was given. These two values were estimated using analytic models and the existing data from the DMSP satellite [31].

The EUSO-Balloon mission provides real data for similar studies for a $\sim 2.5$ hour observation time and $\sim 780 \mathrm{~km}^{2}$. In this section, we show a few preliminary results useful to establish an exposure determination scheme. Further results and discussions can be referred to Reference [14]. EUSO-SPB offered a $\sim 30 \mathrm{~h}$ data with various conditions above the ocean. TUS and Mini-EUSO will allow much deeper analysis of the UV background light.

For the space-based observation of UHECRs, the background light is sorted as extraterrestrial or terrestrial sources, direct or indirect, natural (i.e. astronomical and terrestrial) or artificial light, etc. In the following, major sources of the UV background are summarized together with pathfinders and their pointing directions and altitudes of instruments.

- Extraterrestrial sources [28] (starlight, zodiacal light and temporarily moonlight)

$\downarrow$ (TUS $\sim 500 \mathrm{~km}$; Mini-EUSO $\sim 400 \mathrm{~km}$ )

- Aurora: Transient and regional, mostly in polar regions.

- Airglow emission: Emission from disassociated oxygen atoms by recombining to molecules at $\sim 90 \mathrm{~km}$ altitude. Locational and temporal variations in various scales expected [29, 30].

$\downarrow$ (EUSO-Balloon $\sim 38 \mathrm{~km}$; EUSO-SPB $\sim 16-30 \mathrm{~km}$ )

- Back-scattered light from atmosphere and clouds

$\uparrow$ (Ground-based instrument; EUSO-TA)

- Artificial light: eg. cities

For the nadir-pointing instruments on the balloon, only back-scattered component of the light originating above and the artificial light are observable. In the following, we present a part of the results from EUSO-Balloon that are relevant to the exposure determination [14].

Figure 1 displays temporal variation of the count rates measured by EUSO-Balloon [14]. The vertical axis is the average count rate among the selected pixels. The efficiency of each pixel is normalized to the average.

In earlier part of the time of interest, the large variation and high count rates were observed in the populated and industrial area in Timmins. Around 04:40, EUSO-Balloon was flying above the forest area and presumed to be clear atmosphere conditions. The count rate in this period may be used for a reference $n_{0}$ value in the air shower simulations.

Figure 2 displays the temporal distribution, in form of $\Delta t / \Delta \log n$, of the count rate measured by EUSO-Balloon [14]. The solid histogram denotes the fraction of the time in the 2.5 hour observation time. The dashed curve shows the cumulative fraction. 


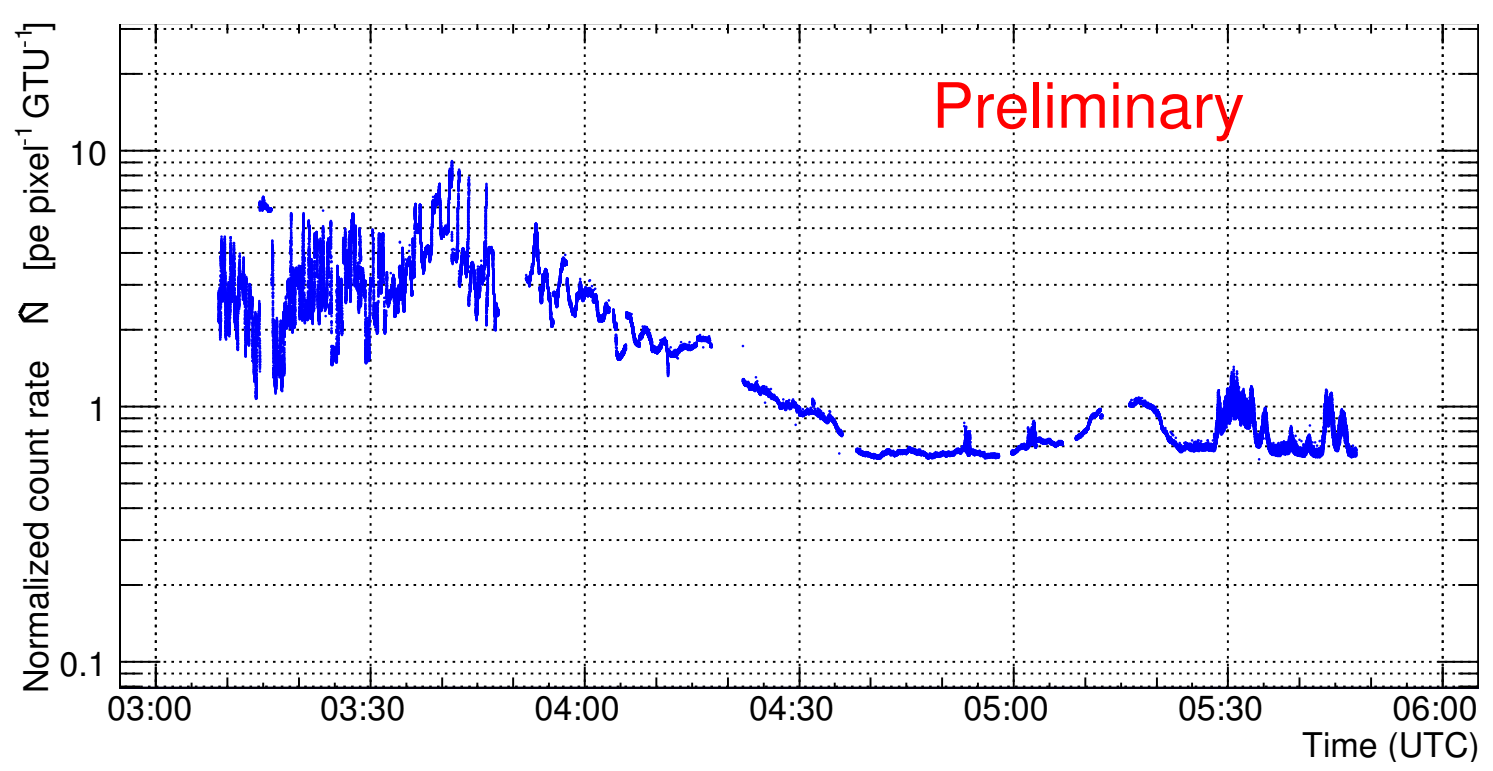

Figure 1: Temporal variation of the count rates measured by EUSO-Balloon [14]. The vertical axis is the average count rate among the selected pixels. The efficiency of each pixel is normalized to the average.

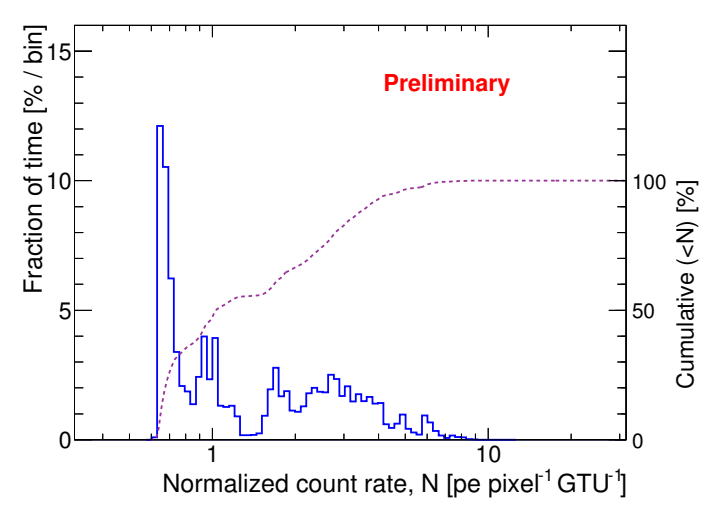

Figure 2: Temporal distribution of the count rates measured by EUSO-Balloon [14]. The cumulative fraction below the given value by the dashed curve to the scale on the right.

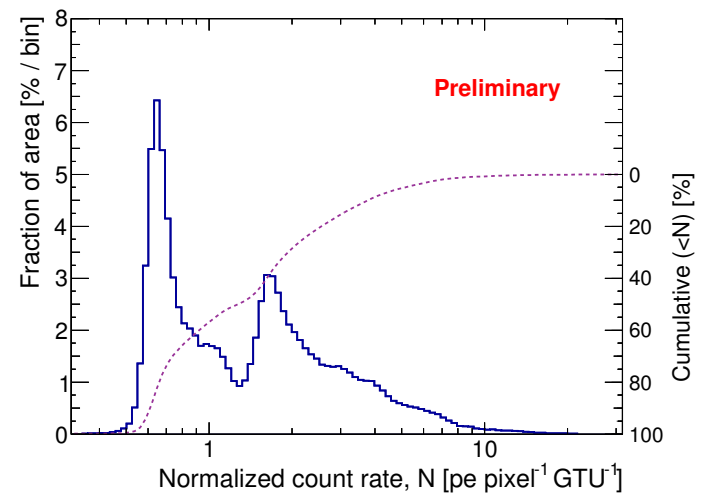

Figure 3: Areal distribution in terms of count rates. The cumulative fraction below the given value by the dashed curve to the scale on the right.

In practice, $\eta$ is determined by the limit $n_{\lim }$ of the count rate allowed by the trigger algorithm is expressed by $\equiv \frac{T_{\mathrm{obs}}}{T_{0}}=\frac{1}{T_{0}} \cdot \int_{0}^{n} \lim \frac{\Delta t}{\Delta n} d n$. From Figure 2, the cumulative fraction in this figure represents this equation. By assuming the lower peak being a result of measurements in the dark forestry area, $\sim 20 \%-30 \%$ of the time was dominated by the diffuse light in the particular time of the observation [14].

Figure 3 displays the areal distribution, in terms of $\Delta s / \Delta \log n$, of count rates in the measured $\sim 780 \mathrm{~km}^{2}$ area by EUSO-Balloon. The dashed curve shows the cumulative fraction above the given value. 
$f_{\text {loc }}$ in Equation (3.6) is given by the cumulative fraction shown in this figure for the Timmins and neighboring area where the EUSO-Balloon measurement was carried out. The higher peak denotes the artificial light sources from industrial facilities such as mines and factories and populated zones. In the full-scale mission that employs more PDMs, the $f_{\text {loc }}$ value represents the fraction of the observation area eliminated from the UEHCR observations.

\section{Summary and outlook}

Using the JEM-EUSO pathfinders on balloons, satellite and on the ground, we have investigated properties of the UV light that acts background sources for the space-based UHECR observations. The EUSO-Balloon data provided a first real data to investigate the exposure determination scheme. Although the validity of the information obtained by EUSO-Balloon is limited for the case of this particular flight, the variation of count rates due to the background light has been well characterized.

For an outlook, the recent EUSO-SPB flight provided $\sim 30 \mathrm{~h}$ data in part together with the infra-red camera [32]. The instantaneous aperture can be estimated by air shower simulations by applying the measured background count rate. Including the cloud impact, namely $\kappa_{C}$ in Equation (3.6), the study for establishing the exposure determination will be promoted using these data.

Other pathfinders, TUS, Mini-EUSO and EUSO-TA also helps improve the knowledge on the background light. The first two mission will cover the large fraction of the Earth and locational and temporal, in various time-scales, dependence of the background light can be more systematically investigated [30]. Mini-EUSO, with a $\sim 400 \mathrm{~km}$ square observation area, will allow to discriminate the impacts by $\eta$ and $f_{\text {low }}$ factors. These pathfinders will allow provide new pieces of information not only for space-based UHECR observations but also for air glow science.

\section{Acknowledgments}

This work was partially supported by Basic Science Interdisciplinary Research Projects of RIKEN and JSPS KAKENHI Grant (22340063, 23340081, and 24244042), by the Italian Ministry of Foreign Affairs and International Cooperation, by the 'Helmholtz Alliance for Astroparticle Physics HAP' funded by the Initiative and Networking Fund of the Helmholtz Association, Germany, by NASA award 11-APRA-0058 in the USA, and by Slovak Academy of Sciences MVTS JEMEUSO as well as VEGA grant agency project 2/0132/17. Russia is supported by the Russian Foundation for Basic Research Grant No 13-02-12175-ofi-m. The Spanish Consortium involved in the JEM-EUSO Space Mission is funded by MICINN \& MINECO under the Space Program projects: AYA2009-06037-E/AYA, AYA-ESP2010-19082, AYA-ESP2011-29489-C03, AYA-ESP2012-39115-C03,AYA-ESP2013-47816-C4, MINECO/FEDER-UNAH13-4E-2741, CSD2009-00064 (Consolider MULTIDARK) and by Comunidad de Madrid (CAM) under projects S2009/ESP-1496 \& S2013/ICE-2822.

\section{References}

[1] The Pierre Auger Observatory (A. Aab et al.), Nucl. Instr. Meth. A798 (2015) 172. 
[2] The Telescope Array Collaboration (R.U. Abbasi et al.), Astrophys. J. Lett. 790 (2014) L21.

[3] H. Sagawa et al., in Proc. of 34th Int. Cosmic Ray Conf. (The Hague; 2015), Pos (ICRC2015) 657.

[4] M. Casolino et al., in these proceedings Pos(ICRC2017) 369.

[5] The JEM-EUSO Collaboration (J.H. Adams Jr. et. al.), Exp. Astron. 40 (2015) 3.

[6] M. Casolino et al., in these proceedings Pos(ICRC2017) 368.

[7] The JEM-EUSO Collaboration (J.H. Adams Jr. et. al.), Exp. Astron. 40 (2015) 19.

[8] L. Tkachev et al., in these proceedings Pos(ICRC2017) 527.

[9] The JEM-EUSO Collaboration (J.H. Adams Jr. et. al.), Exp. Astron. 40 (2015) 315.

[10] P.A. Klimov et al., arxiv:1706.04976.

[11] M. Ricci et al., in these proceedings Pos(ICRC2017) 440.

[12] The JEM-EUSO Collaboration (J.H. Adams Jr. et. al.), Exp. Astron. 40 (2015) 61.

[13] S. Mackovjack et al., in Proc. of 34th Int. Cosmic Ray Conf. (The Hague; 2015) Pos (ICRC2015) 685.

[14] G. Abdellaoui et al. (in preparation)

[15] M. Bertaina et al., in these proceedings Pos(ICRC2017) 445.

[16] L. Wiencke et al., highlight talk in the Conference [CRI306].

[17] NASA Balloon Program Office; 'NASA Code 820 (Super Pressure Balloon)'. http://sites.wff.nasa.gov/code820/spb.html

[18] G. Abellaoui et al., Nucl. Instr. Meth. A, 866 (2017) 150.

[19] J. Bayer et al., in these proceedings Pos(ICRC2017) 445.

[20] F. Fenu et al., in these proceedings, Pos(ICRC2017) 486.

[21] The JEM-EUSO Collaboration (J.H. Adams Jr. et. al.), Exp. Astron. 40 (2015) 301.

[22] L.W. Piotrowski et al., in these proceedings, Pos(ICRC2017) 374.

[23] F. Bisconti et al., in these proceedings, Pos(ICRC2017) 463.

[24] The JEM-EUSO Collaboration (J.H. Adams Jr. et. al.), Exp. Astron. 40 (2015) 117.

[25] J. Bayer et al., in Proc. of 33rd Int. Cosmic Ray Conf. (Rio de Janeiro; 2013) ID0432.

[26] J.H. Adams Jr.et al., Astropart. Phys. 44 (2013) 76.

[27] The JEM-EUSO Collaboration (J.H. Adams Jr. et. al.), Exp. Astron. 40 (2015) 135.

[28] C. Leinert et al., Astron. Astrophys. Suppl. Ser. 127 (1998) 1.

[29] V.Y. Khomich, A.I. Semenov and N.N. Shefov, "Airglow as an Indicator of Upper Atmospheric Structure and Dynamics”, Springer-Verlag GmbH, Heidelberg (2008) ISBN 978-3-540-75833.

[30] P. Bobik et al., in these proceedings, Pos(ICRC2017) 408.

[31] NOAA National Center for Environmental Information; 'DMSP (Defense Meteorological Satellite Program) data'. http://ngdc.noaa.gov/eog/dmsp.html

[32] A. Allen et al., in these proceedings, Pos(ICRC2017) 436. 